Programa de Estudios de Posgrado en Geografía E P G

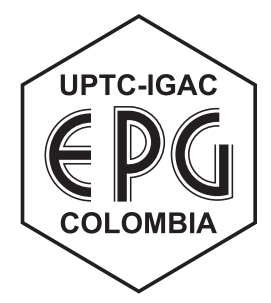

\title{
PERSPECTIVA GEOGRÁFICA
}

\author{
Volumen 21 No. 1, 2016 \\ Enero - Junio
}

\section{PERSPECTIVA GEOGRÁFICA}

Perspect. geogr. - Tunja (Colombia) Vol. 21 No. 1, 2016 enero - junio ISSN 01 23-3769 (Impreso) - 2500-8684 (En línea) 


\title{
Perspectiva Geográfica
}

Es una revista académica arbitrada, de periodicidad semestral desde el 2013, especializada en estudios geográficos y de planificación territorial, dirigida a comunidades académicas, investigativas y del desarrollo territorial, interesadas en temas geográficos y ciencias afines. La publicación es editada por el programa de Estudios de Posgrado en Geografía -EPG- en el marco del convenio de colaboración científica entre la Universidad Pedagógica y Tecnológica de Colombia -UPTC- y el Instituto Geográfico Agustín Codazzi -IGAC- desde 1987. En la revista se publican artículos originales e inéditos de investigación, reflexión y revisión, referidos a la espacialidad de los fenómenos sociales, económicos, políticos, culturales y naturales, desde diversas perspectivas y enfoques teóricos. También acoge los estudios sobre aspectos técnicos y metodológicos del quehacer propio de los geógrafos, así como los trabajos de geografía aplicada en los campos de la geomática, la planificación territorial y las investigaciones de frontera.

Indexada en:

Índice Bibliográfico Nacional -PUBLINDEX- Dpto. Administrativo de Ciencia, Tecnología e Innovación, Colciencias Citas Latinoamericanas en Ciencias Sociales y Humanidades de la UNAM -CLASE Sistema de Información Sobre las Revistas de Investigación Científica -LATINDEX

De consulta en la plataforma de recursos y servicios documentales:

UPTC: http://www.uptc.edu.co/enlaces/rpersgeogra

DIALNET: http://dialnet.unirioja.es/servlet/revista?codigo=15743

Perspectiva geográfica / Programa de Estudios de Posgrado en Geografía EPG, Convenio UPTC - IGAC. - No. 1 (1997)- Tunja: UPTC, IGAC, 1997- ISSN 0123-3769 (Impreso) - 2500-8684 (En línea)

Semestral 1997-2003 - Anual desde 2004. A partir de 2008 se convierte a volumen 1. Periodicidad Semestral desde el primer semestre de 2013. Geografía Física - Publicaciones seriadas. - 2. Geografía humana - Publicaciones seriadas. - 3. Geografía humana - Colombia - Publicaciones seriadas - 4. Uptc - Publicaciones seriadas $\operatorname{CDD} 900$

Correspondencia:

Editor: Rigaud Sanabria Marin

Carrera 30 Núm. 48-51, Edificio CIAF

Oficina 108, Bogotá (Colombia)

Teléfono: (57-1) 3694000 / 4100 Ext. 91631

Telefax: (57-1) 3694080

perspectiva.geografica@uptc.edu.co

Canje:

\author{
Universidad Pedagógica y Tecnológica de Colombia (UPTC) \\ BIBLIOTECA CENTRAL \\ Avenida Central del Norte \\ Tunja-Boyacá-Colombia \\ biblioteca.informacion@uptc.edu.co
}

Editora en Jefe UPTC: Ruth Nayibe Cárdenas Soler

Corrección de estilo: Luis Enrique Clavijo Morales

Corrección abstract: Alexánder Ortiz Carvajal, Instituto Internacional de Idiomas, UPTC

Diagramación: Lorena Orduz Valbuena, Diseñadora Gráfica, Búhos Editores.

Diseño de portadas: Oficina de Difusión y Mercadeo, IGAC

Retoque gráficos: Lorena Orduz Valbuena, Diseñadora Gráfica, Búhos Editores.

llustración en portada: Banco de Imágenes, CIAF-IGAC

Impresión:

BÚHOS EDITORES LTDA.

Diagonal 57 No. 7 - 34

Barrio Santa Ana

Tunja, Boyacá (Colombia)

buhosedi@gmail.com

Tel. $7442264 \cdot$ Cel. 3144115024
Tamaño $17.5 \times 25 \mathrm{cms}$

Impresa en Papel propalmate de 90 gramos. Carátula impresa en papel propalcote de 300 gramos, en policromía.

Publicación financiada por la Dirección de Investigaciones y el Programa de Estudios de Posgrado en Geografía.

Las opiniones expresadas en los artículos son de exclusiva responsabilidad de sus autores.

Se permite la reproducción parcial o total citando siempre la fuente.

Esta revista se terminó de imprimir en Búhos Editores Ltda., con una edición de 400 ejemplares. Tunja-Boyacá-Colombia 


\section{Programa de Estudios de Posgrado en Geografía, EPG}

Convenio Universidad Pedagógica y Tecnológica de Colombia, UPTC Instituto Geográfico Agustín Codazzi, IGAC

\section{Consejo Directivo}

GUSTAVO ORLANDO ÁLVAREZ ÁLVAREZ, Esp.

Rector, Universidad Pedagógica y Tecnológica de Colombia (UPTC)

JUAN ANTONIO NIETO ESCALANTE, M.SC.

Director General

Instituto Geográfico Agustín Codazzi (IGAC)

OLGANAJAR SÁNCHEZ, M.SC.

Decana Facultad de Ciencias de la Educación (UPTC)

HÉCTOR MAURICIO RAMÍREZ DAZA, M.SC.

Jefe, Oficina CIAF

Instituto Geográfico Agustín Codazzi (IGAC)

Perspectiva Geográfica, Volumen 21 No. 1, ISSN 0123-3769 (Impreso) - 2500-8684 (En línea) - Año 2016 enero - junio Publicación coordinada por el Programa de Estudios de Posgrado en Geografía (EPG), Convenio UPTC-IGAC

\section{Editor:}

RIGAUD SANABRIA MARIN, Ph.D.

\section{Asistentes Editoriales:}

JORGE LEONARDO ROJAS CORREDOR, Lic.

JULY ALEJANDRA CASTRO HERNÁNDEZ, Filósofa.

\section{Comité Editorial:}

ÁNGEL MASSIRIS CABEZA, Ph.D.

Consultor Internacional

(Colombia)

DELFINA TRINCA FIGHERA, Ph.D.

Universidad de los Andes

(Venezuela)

EDUARDO SALINAS CHÁVES, Ph.D.

Universidad de La Habana

(Cuba)

EFRAIIN LLANOS HENRIQUEZ, Ph.D.

Universidad del Atlántico

(Colombia)

FRANZ GUTIÉRREZ REY, Ph.D.

Universidad Pedagógica y Tecnológica de Colombia

(Colombia)

HÉCTOR MAURICIO RAMÍREZ DAZA, M.Sc.

Instituto Geográfico Agustín Codazzi, IGAC

(Colombia)

JOSÉ GÓMEZ ZOTANO, Ph.D.

Universidad de Granada

(España)

ÓSCAR BUITRAGO BERMÚDEZ, Ph.D

Universidad del Vallle

(Colombia)

\section{Coordinadores académicos EPG}

FRANZ GUTIÉRREZ REY, Ph.D.

Maestría en Geografia

RIGAUD SANABRIA MARIN, Ph.D.

Doctorado en Geografía

\section{Comité Científico:}

DAVID VELÁSQUEZ TORRES, Ph.D.

Universidad de Quintana Roo

(México)

ELKIN VELÁSQUEZ MONSALVEC, Ph.D.

Jefe de la Sección de Gobernanza Urbana de la ONU - Habitat (Colombia)

JAIR PRECIADO BELTRÁN, Ph.D.

Universidad Distrital Francisco José de Caldas

(Colombia)

JOAQUÍN FARINOS DASI, Ph.D.

Universidad de Valencia (España)

LUIS CHÍAS BECERRIL, Ph.D.

Universidad Nacional Autónoma de México (México)

MARÍA ELINA GUDIÑO DE MUÑOZ, Ph.D.

Universidad Nacional de Cuyo

(Argentina)

ROBERTO GONZÁLEZ SOUSA, Ph.D.

Universidad de La Habana

(Cuba) 
El comité editorial agradece a los jurados que hicieron posible este volumen:

ANNA ORTIZ GUITART, Ph.D.

Universitad Autónoma de Barcelona

(España)

BELÉN ELISA DÍAZ PÉREZ, Ph.D.

Universidad Rey Juan Carlos

(España)

CARLOS DANIEL VERGARA CONSTELA, M.SC.

Universidad de Valparaíso

(Chile)

CLEMENCIA ESCALLÓN GARTNER, M.Sc.

Universidad de los Andes

(Colombia)

DANIEL FRANCISCO JARAMILLO JARAMILLO, M.SC.

Universidad Nacional de Colombia

(Colombia)

DIANA BURGOS VIGNA, Ph.D.

Université de Cergy-Pontoise

(Francia)

GLORIA CECILIA RUALES ZAMBRANO, M.Sc (Cand.)

Universidad Nacional Abierta y a Distancia

(Colombia)

GLORIA YANETH FLÓREZ YEPEZ, M.Sc.

Universidad de Manizales

(Colombia)

INMACULADA CARAVACA BARROSO, Ph.D.

Universidad de Sevilla

(España)

ISAÍAS TOBASURA ACUÑA, Ph.D.

Universidad de Caldas

(Colombia)

JORGE ALIRIO ARISTIZABAL BOTERO, M.Sc.

Universidad EAFIT

(Colombia)

JOSÉ LUIS VARA MUÑOZ, Ph.D.

Universidad Autónoma de Madrid

(España)

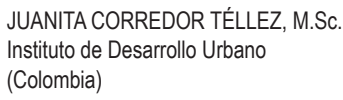

MARGARITA MARÍA RODRÍGUEZ GARCÉS, M.SC. Consultora independiente

(Colombia)

MARÍA CRISTINA ORDOÑEZ DÍAZ, Ph.D. (Cand.)

Proyecto SGR Cauca

(Colombia)

MARTHA CONSTANZA DAZA TORRES, Ph.D. (Cand.) Universidad del Valle

(Colombia)

NANCY LEIVA GUTIÉRREZ, M.Sc.

Instituto Geográfico Agustín Codazzi

(Colombia)

OMAR URAN ALONSO ARENAS, Ph.D.

Universidad de Antioquia

(Colombia)

PEDRO LEÓN GARCÍA REINOSO, Ph.D. (Cand.)

Universidad del Quindio

(Colombia)

PILAR DÍAZ FORERO, Esp.

Universidad Piloto de Colombia

(Colombia)

SAMUEL DARÍO GUZMÁN LÓPEZ, Ph.D. (Cand.)

Universidad Tecnológica de Pereira

(Colombia)

WILLIAM HERNANDO ALFONSO PIÑA, Ph.D.

Universidad del Rosario

(Colombia)

WILSON VERGARA VERGARA, M.Sc.

Universidad de la Salle

(Colombia) 


\section{Contenido}

\section{Content}

Presentación

Presentation

La conformación del espacio social a través del gusto por lo pintoresco

Social Space Conformation for the Sake of the Picturesque Augusto Solórzano

Modelo climático de los páramos de la cordillera Oriental colombiana aplicado a regímenes de temperatura del suelo

Climate Model of the Moorlands of the Colombian Eastern Mountain Range Applied to Soil Temperature Regimes

Carlos Enrique Castro Méndez, Yolima del Carmen Agualimpia Dualiby y

Fernando Sánchez Sánchez 33

Análisis de la distribución espacial y temporal de la precipitación en la cuenca del río Chicú, Sabana de Bogotá, Colombia

Spatial and Temporal Precipitation Distribution Analysis at Chicú River Basin, Bogotá s High Altitude Savannah, Colombia

Cristian Camilo López Velandia

Potencial de captura de $\mathrm{CO}_{2}$ asociado al componente edáfico en páramos Guantiva-La Rusia, departamento de Boyacá, Colombia

Potential $\mathrm{CO}_{2}$ Capture Associated with Edaphic Component in Moorlands Guantiva-La Rusia, Department of Boyacá, Colombia

Karen Lisseth Africano Pérez, Germán Eduardo Cely Reyes y

Pablo Antonio Serrano Cely

Estructura agraria: lógicas disimiles en la concepción del territorio

Agrarian Structure: Different Fields of Logic on the Concept of Territory

Wilson González Santos 
Aproximación a la gestión del ordenamiento territorial en Colombia frente a los megaproyectos de infraestructura

Approach to the Management of Land Use in Colombia Against Large Infrastructure Projects Jahir A. Gutiérrez O., Gleidy A. Urrego E. y Jefferson Carmona M.

Análisis geo-histórico del proceso de urbanización en América del Sur: De la ciudad indoamericana a la ciudad neoliberal

Geo-Historical Analysis of the Urbanization Process in South America: From the Pre-Columbian to the Modern Neoliberal Period

Jorge Andrés Rivera Pabón

Guía para autores

Guidelines for Authors 


\section{Presentación}

Presentation

En el 2016 continuamos, con agrado y entusiasmo, presentando los importantes resultados de estudios geográficos y de planificación territorial, de acuerdo con nuestra misión de divulgarlos en la comunidad académica, investigativa y de la planificación territorial y al público en general interesado en profundizar en la actualidad de las estructuras y dinámicas territoriales.

En este el primer número del año, nuestros autores nos comparten resultados de sus recientes investigaciones referentes a diferentes enfoques, desde la geografía de las emociones, la geografía física, la geografía agraria, la geografía histórica, la geografía urbana y el ordenamiento territorial.

Augusto Solórzano analiza la importancia de las experiencias cotidianas y las percepciones afectivas de lo pintoresco en la construcción del espacio geográfico por la sociedad y en hacer de su territorio un lugar de goce, denominado paisaje emocional, en el cual se elabora la experiencia humana.

Carlos Enrique Castro, Yolima del Carmen Agualimpia y Fernando Sánchez proponen un modelo climático que permite determinar los regímenes de temperatura del suelo en ecosistemas de páramo, a partir de la relación de temperatura del aire y del suelo, el aspecto de la vertiente y la diferencia de gradientes de temperatura y altitud, para facilitar la generación de cartografía semidetallada de isotermas. 
Cristian Camilo López analiza la distribución de precipitaciones a escala local, para la cuenca del río Chicú en Colombia, encontrando un aumento sostenido de las precipitaciones en las dos últimas décadas, con importante variabilidad espacial entre los mínimos y máximos, aumentando los valores hacia la cuenca baja. Evidencia el autor la disminución de la precipitación en periodos El Niño y el correspondiente aumento en periodos La Niña.

Karen Lisseth Africano, Germán Eduardo Cely y Pablo Antonio Serrano evidencian el importante impacto que tiene en la mitigación del cambio climático la captura de carbono por los suelos de los páramos Guantiva La Rusia. Encuentran los autores, para este importante territorio, que la captura de carbono es significativamente mayor en suelos en recuperación; menor en suelos no intervenidos, y significativamente menor en los suelos de páramo con intervención antrópica.

Wilson González parte del análisis del conflicto y del desarrollo de la estructura agraria para proponer la trialéctica espacial en la comprensión del campesinado y sus dinámicas socioeconómicas, incluyéndole al enfoque tradicional bimodal la interacción espacial de los procesos de intervención rural en la comprensión de la construcción social del territorio campesino.

Jahir Gutiérrez, Gleidy Urrego y Jefferson Carmona analizan el marco jurídico del ordenamiento territorial y su incompatibilidad en los diferentes niveles territoriales en Colombia, así como la desarticulación de las entidades encargadas de la gestión estatal de megaproyectos. A partir de la interpretación de los diversos elementos y actores del territorio involucrados en los megaproyectos, plantean los autores que el ordenamiento territorial nacional, articulado con los ordenamientos territoriales departamentales y municipales, es necesario para resolver los problemas relacionados con la disposición, ocupación y uso del suelo por los megaproyectos de infraestructura.

Jorge Andrés Rivera Pabón, considerando, principalmente, el enfoque materialista histórico geográfico, analiza la estructura del poblamiento y las dinámicas urbanas, desde el periodo precolombino al actual, para comprender el proceso de urbanización en América del Sur. Encuentra entre los elementos sobresalientes el carácter centrípeto y la localización interior del periodo 
precolombino, el carácter periférico y litoral del periodo colonial, el carácter desconcentrado e interior en el periodo moderno, y el carácter de grandes ciudades en el periodo posmoderno.

Nuevamente, esta publicación de Perspectiva Geográfica es resultado de un gran número de colaboradores que, proactivamente, siempre están dispuestos a contribuir con el desarrollo y divulgación de la productividad geográfica, como son los autores, los árbitros, los miembros del comité científico y editorial, los correctores de estilo, los diagramadores e impresores, los integrantes del equipo editorial, así como las directivas y funcionarios de la Universidad Pedagógica y Tecnológica de Colombia (Uptc) y del Instituto Geográfico Agustín Codazzi (IGAC); a todos, un profundo agradecimiento.

Invitamos a todos los interesados en las últimas investigaciones geográficas de nuestros territorios a consultar los diferentes números de la revista, que se encuentran publicados en la página Web de la Uptc, así como en las bases de datos registradas.

Rigaud Sanabria Marin

Editor 